\section{Développement, migrations et gradients}

Une des clés du développement est que les cellules dans l'organisme et les protéines dans les cellules se trouvent à leur place normale. La mutation reeler perturbe typiquement la migration cellulaire, en l'occurrence celle des neurones corticaux qui ne s'arrêtent pas au cours de leur migration, dépassent leur position attitrée et se pressent au-delà, en périphérie de leur champ de migration. Le produit du gène reelin semble être une protéine de la matrice extracellulaire qui est sécrétée par des cellules ayant migré précocement en périphérie et forme un gradient répulsif essentiel à l'arrêt de la migration des neuromes migrant ultérieurement. C'est aussi une interaction inhibitrice qui explique, dans l'embryon syncytial de drosophile, le gradient croissant de distribution antéro-postérieur de la protéine Caudal, dont le messager est distribué de façon homogène: sa traduction est inhibée par la protéine Bicoid, distribuée en gradient à partir d'un site de synthèse au pôle antérieur où se trouve concentré l'ARNm. Nous rapportons aussi un nouvel exemple de redondance fonctionnelle entre les membres d'une famille de protéines inductrices $d u$ développement, en l'occurrence les facteurs myogéniques de la famille MyoD: les protéines Myf5 et myogénine sont parfaitement interchangeables, la spécificité fonctionnelle de leurs gènes respectifs étant due à leurs différences d'expression spatiotemporelle. Ces résultats ont été obtenus par recombinaison homologue dans des cellules souches embryonnaires ES, méthode qui ne fonctionnait jusqu'alors que chez la souris. Mais, peut-être, d'autres espèces pourraient se prêter dans le futur à ces techniques, par exemple les brebis.

\title{
Mutation reeler et protéine reelin : bases d'un nouveau modèle du développement cortical
}

Reeler est une mutation récessive autosomique de la souris, qui affecte le développement embryonnaire du cerveau d'une manière tout à fait particulière. Chez l'embryon normal, comme résumé sur la figure 1, les neurones corticaux sont engendrés le long des ventricules, au sein des zones ventriculaires. Les éléments postmitotiques migrent ensuite radialement en direction des méninges, en intime relation avec des fibres gliales radiaires. Les premiers neurones engendrés ont une organisation horizontale et deviennent les cellules de Cajal-Retzius et les neurones de la sous-plaque. Les neurones de la plaque corticale sont engendrés un peu plus tard et ont une organisation radiaire très précise. Chez l'embryon reeler, les neurones sont engendrés en nombre et selon une chronologie normale, et migrent le long des fibres gliales radiaires comme chez les embryons normaux [1, 2]. Cependant, arrivés près de leur destination, les neurones en migration se comportent comme s'ils avaient perdu la capacité de s'ordonner selon les agencements architectoniques normaux. Les cellules de la sous-plaque sont comme repoussées vers l'extérieur par le développement d'une plaque corticale dont l'organisation radiaire est grossièrement défectueuse. Il en résulte une malformation cérébrale spectaculaire. Au niveau du cortex, les couches sont mal séparées et la maturation se produit de l'extérieur vers l'intérieur, selon un gradient inversé par rapport au cortex normal [2-5]. Le cortex n'est pas la seule cible de la mutation qui perturbe le développement architectonique précoce de nombreuses structures du système nerveux central $[6,7]$.

Début 1995, un candidat pour le gène reeler fut cloné a partir d'un nouvel allèle engendré fortuitement par insertion transgénique. Ce candidat, appelé reelin [8], fut aussi identifié par clonage positionnel [9, 10]. L'ARNm codé par le gène reelin est de grande taille (environ 12000 bases) et possède une phase ouverte de lecture de 10383 bases correspondant à une protéine de 3461 résidus. La séquence débute par un segment hydrophobe, probablement un peptide signal. Ensuite, on trouve successivement un segment similaire $(\sim 25 \%)$ à la F-spondine, une séquence anonyme, puis une série de 8 répétitions de 350 à 390 résidus. Chacune de ces répétitions est composée de deux domaines de taille à peu près identique, séparés par un motif EGF similaire à ceux de la ténascine et de l'intégrine $\beta$. La protéine se termine par un court segment riche en arginine et donc positivement chargé. A part les similitudes mentionnées, la reelin ne ressemble à aucune protéine décrite. La séquence de l'ADNc suggère que la reelin est une protéine sécrétée dans la matrice extracellulaire, une hypothèse renforcée par la présence de sites potentiels de glycanation (O-xylosylation), de liaison à l'héparine et d'autres modifications post-traductionnelles.

L'ARNm de la reelin peut être détecté dans le cerveau embryonnaire de souris à partir du 12e jour (E12). Sa concentration augmente au cours du développement pour être maximale autour de la naissance et la période 


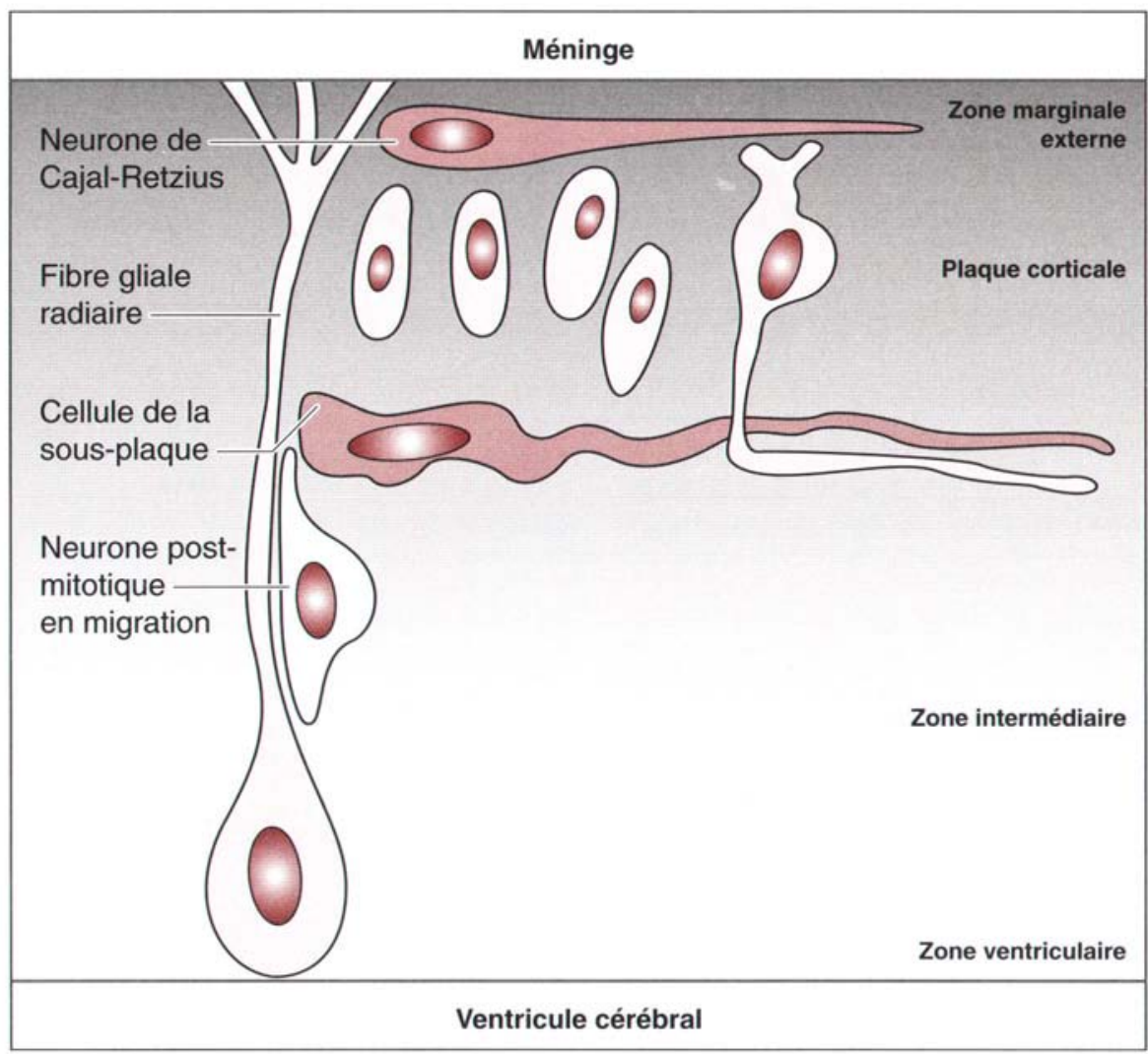

périnatale, puis diminue progressivement et persiste à bas bruit dans le cerveau adulte. Les premières analyses par hybridation in situ démontrent une expression extrêmement forte dans des cellules localisées à la périphérie de la zone marginale du télencéphale embryonnaire, qui correspondent aux neurones de CajalRetzius [8] ; un exemple est montré sur la figure 2. Il existe aussi une forte expression dans le bulbe olfactif, au niveau des futures cellules mitrales. Aucun signal significatif n'est détecté dans la plaque corticale embryonnaire, ce qui est quelque peu surprenant. En revanche, une expression modérée s'installe au niveau de neurones localisés dans les couches profondes du cortex vers la naissance et y persiste durant la période postnatale de maturation. D'autres structures du système nerveux central sont caractérisées par une expression variable du messager de la reelin. Par exemple, un signal d'intensité moyenne est détecté dans la partie interne de la couche granulaire externe du cerve- couche granulaire interne pendant la période de maturation. Aux stades embryonnaires, la reelin est aussi synthétisée dans la corne antérieure de la moelle, au niveau de la partie centrale du tectum mésencéphalique (futurs tubercules quadrijumeaux), au niveau d'un petit noyau du tegmentum mésencéphalique qui pourrait correspondre au locus coeruleus, au niveau des neurones rétiniens et dans quelques autres structures du système nerveux central. Durant la période postnatale, l'expression de l'ARNm de la reelin est aussi perçue dans de nombreuses structures limbiques telles que les noyaux du septum ou l'aire préoptique.

La production d'anticorps anti-reelin n'a pas encore abouti. Cependant, il est très probable que l'anticorps monoclonal CR-50 [11] soit dirigé contre un épitope de la reelin. Cet anticorps monoclonal a été obtenu en immunisant des souris reeler avec du cerveau embryonnaire de souris normale. Il colore spécifiquement les cellules de Cajal-Retzius et n'est pas détectable dans le cerveau embryon-
Figure 1. Modèle de l'action de la reelin au niveau du cortex embryonnaire normal. Le dessin représente une coupe au niveau du cortex de souris au quatorzième jour embryonnaire. Le ventricule est en bas et la méninge en haut. Les neurones postmitotiques, engendrés dans la zone ventriculaire migrent radialement à travers la zone intermédiaire, le long des fibres gliales radiaires qui forment un écheveau radial fin et participent à la formation de la limitante externe. L'organisation horizontale primitive comprend les neurones de Cajal-Retzius, situés dans la zone marginale externe, et les cellules de la sous-plaque. Ces deux contingents sont séparés par la plaque corticale dont les neurones ont une organisation radiaire. La reelin sécrétée par les neurones de Cajal-Retzius formerait un gradient (représenté comme le dégradé de gris) répulsif pour les neurones de la plaque corticale. Le réseau répulsif de reelin maintiendrait les cellules de la plaque corticale à distance de la limitante externe et serait ainsi indispensable à la formation de la zone marginale, ainsi qu'au maintien de l'intégrité des prolongements externes des cellules radiaires.

naire reeler (allèle $r l$ classique). Au moyen de cet anticorps CR-50, nous avons montré que les cellules de Cajal-Retzius sont bien colorées dans le cerveau des souris reeler de l'allèle Orléans (figure 1). Cette mutation $\mathrm{rl}^{\mathrm{Orl}}$ est caractérisée par la délétion d'un exon du messager de la reelin [9], qui entraîne un cadre de lecture tronqué et probablement la production d'une protéine tronquée. Nos observations avec l'anticorps CR-50 suggèrent donc fortement que l'épitope reconnu est associé à la reelin. De plus, elles confirment que les cellules de Cajal-Retzius sont présentes chez les souris reeler et que la mutation n'affecte ni leur position ni leur différenciation.

Les premiers résultats concernant l'étude de la distribution de la reelin dans le cerveau embryonnaire font apparaître un paradoxe apparent puisque des cellules qui expriment le transcrit et probablement la protéine reelin (pour autant que l'anticorps CR-50 soit bien dirigé contre cette protéine), telles que les cellules de Cajal-Retzius, sont disposées à peu 

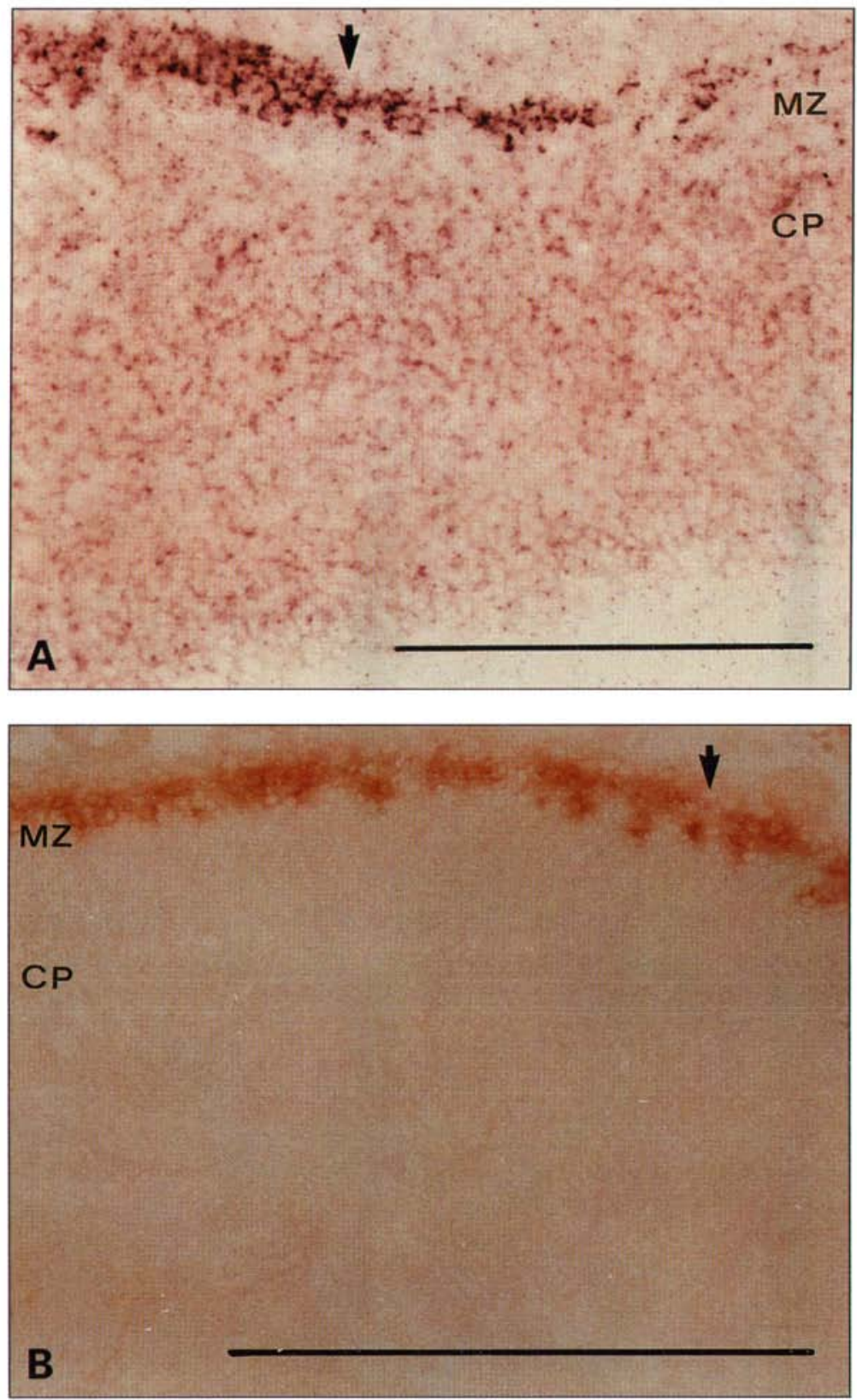

Figure 2. Mise en évidence du messager de la reelin chez un embryon de souris. A. embryon normal âgé de 16 jours. Coupe coronale au niveau du télencéphale médian (primordium hippocampique). Hybridation in situ avec une sonde antisens correspondant au messager de la reelin (système digoxigénine de Boehringer). L'expression du messager est, à ce stade, restreinte aux neurones de Cajal-Retzius (flèche). B. Embryon de souris âgé de 18 jours, homozygote pour la mutation reeler, allèle Orléans. Coupe coronale au niveau du cortex. La coloration par immunoperoxidase (substrat DAB) au moyen de l'anticorps CR-50 [11] met en évidence les cellules de Cajal-Retzius (flèche) à leur place normale, dans la zone marginale (MZ) chez l'embryon reeler. CP: plaque corticale. Barre: $200 \mu \mathrm{m}$. près normalement dans le cerveau embryonnaire reeler, alors que d'autres éléments, en particulier les cellules de la plaque corticale, qui sont très anormalement ordonnées chez le mutant, n'expriment pas le gène reelin. Une solution évidente de ce paradoxe consiste à postuler une action indirecte des cellules de CajalRetzius sur le développement de la plaque corticale. En d'autres termes, les neurones de Cajal-Retzius, via la sécrétion de reelin dans la matrice extracellulaire locale de la zone marginale, influenceraient le développement du cortex en agissant sur les neurones de la plaque corticale et/ou sur l'écheveau des fibres gliales radiaires qui guident la migration neuronale [12, 13]. Plusieurs modèles sont, bien sûr, envisageables. Une hypothèse qui nous paraît intéressante, fondée sur une suggestion de A. Pearlman (communication personnelle), est que la reelin forme, dans la matrice extracellulaire de la zone marginale, un réseau répulsif pour les neurones en migration. Cette action répulsive pourrait résulter par exemple d'un rayon hydrodynamique élevé, un mécanisme analogue à celui proposé par Rutishauser pour expliquer la distribution et l'action de la forme polysialylée de la N-CAM [14]. En repoussant les neurones de la plaque corticale, ce réseau de reelin participerait à la formation d'une zone marginale peu cellulaire et, indirectement, à l'organisation laminaire de la plaque corticale précoce. De même, une zone marginale normale serait nécessaire au maintien de l'intégrité des pieds externes des fibres gliales radiaires [19], qui ont une morphologie très délicate. Quelques observations plaident en faveur de cette hypothèse. Derer et al. [15] ont démontré que les prolongements externes des cellules gliales radiaires sont altérés chez les souris reeler. Les mêmes auteurs ont également proposé que la matrice extracellulaire et les cellules de Cajal-Retzius jouent un rôle important dans l'organisation de la plaque corticale chez l'animal normal et dans sa perturbation chez la souris reeler [16-18]. De plus, une étude récente suggère que l'ablation des 
cellules de Cajal-Retzius semble perturber l'écheveau glial et la migration neuronale radiaire [20].

Un mécanisme analogue pourrait expliquer l'action de la reelin sur le cervelet embryonnaire. Les cellules granulaires externes joueraient un rôle équivalent à celui des cellules de Cajal-Retzius au niveau du télencéphale et influenceraient la formation de la plaque des cellules de Purkinje par la formation d'un réseau répulsif de reelin dans la zone marginale. De même, une action répulsive de la reelin cadre bien avec le profil d'expression observé à d'autres niveaux de l'encéphale ou à des stades plus tardifs du développement. Par exemple, la sécrétion de reelin par quelques cellules de la profondeur du cortex pendant la période postnatale pourrait contribuer à la séparation des corps cellulaires bien visible morphologiquement à ce stade et, partant, faciliter l'ascension dans le cortex des fibres thalamiques qui patientent dans le sous-cortex depuis les stades embryonnaires tardifs (pour une discussion du développement des afférences thalamo-corticales voir [21]). En revanche, nous ne voyons pas actuellement comment expliquer les anomalies présentes au niveau de plusieurs structures du tronc cérébral, en particulier l'olive inférieure. Nous avons montré voici plusieurs années que l'olive bulbaire se développe anormalement chez les souris reeler [5]. Or nous ne sommes pas parvenus jusqu'ici à mettre en évidence le messager reelin dans cette structure, ni au niveau des cellules gliales qui l'entourent. De même, nous n'avons obtenu aucune coloration au niveau de l'olive inférieure par l'anticorps CR-50. La même remarque peut être faite concernant d'autres noyaux du tronc cérébral, comme le noyau du nerf facial qui est, lui aussi, fortement affecté par la mutation [6].

En conclusion, le remarquable travail de D'Arcangelo et al. [8] nous donne enfin l'occasion de comprendre l'action de la mutation reeler et ainsi de clarifier un mécanisme clé du développement cérébral. Comme tout travail majeur, le clonage de l'ADNc de nouvelles questions et procurera, à n'en pas douter, matière à réflexion pour quelques années encore

\section{André M. Goffinet}

Département de physiologie humaine, Faculté de Médecine, Facultés Universitaires Notre-Dame de la Paix, 61, rue de Bruxelles, B-5000 Namur, Belgique.

\section{Remerciements}

Mes remerciements s'adressent à tous les membres de mon laboratoire qui collaborent à l'étude de la mutation reeler, ainsi qu'au Dr.S. Schiffman (Université Libre de Bruxelles) qui participe à l'étude du messager de la reelin par hybridation in situ. Je remercie aussi le Dr. A. Pearlman pour sa discussion d'un rôle répulsif possible de la reelin.

\section{* GLOSSAIRE}

Ténascine: protéine de la matrice extracellulaire, souvent associée à la fibronectine, en particulier dans les tissus conjonctifs, mais également présente dans le cerveau embryonnaire. Auparavant également appelée cytotactine, hexabrachion, ou 51 [21].

F-spondine: protéine de la matrice extracellulaire possédant une analogie avec la thrombospondine et synthétisée par la lame basale (floor plate) $d u$ tube neural embryonnaire. Elle interviendrait dans le développement de la moelle [22].

O-xylosylation: attachement principal des glycosaminoglycans sur les protéines axiales des protéoglycans, par fixation d'un résidu xylose sur la sérine, au niveau d'un site consensus SGXG (S: sérine; G: glycine; $X$ : résidu quelconque).

\section{TIRÉS À PART}

A.M. Goffinet.

\section{RÉFÉRENCES}

1. Rakic P. Mode of cell migration to the superficial layers of fetal monkey neocortex. J Comp Neurol 1972 ; 145 : 61-84.

2. Caviness VS Jr, Rakic P. Mechanisms of cortical development: A view from mutations in mice. Annu Rev Neurosci 1978 ; 1 : 297-326.

3. Rakic P. Neurons in rhesus monkey visual cortex: systematic relation between time of origin and eventual disposition. Science $1974 ; 183: 425-7$

4. Caviness VS, Jr. Neocortical histogenesis in normal and reeler mice: A developmental study based upon $\left[{ }^{3} \mathrm{H}\right]$ thymidine autoradiography. Dev Brain Res 1982 ; 4 : 293-302.

5. Peschanski M. Le développement radiaire du cortex cérébral remis en cause. In: Peschanski M, ed. Lexique neurobiologie. Paris : John Libbey Eurotext, 1992 : 47-9.

6. Goffinet AM. Events governing organization of postmigratory neurons: studies on brain development in normal and reeler mice. Brain Res Rev 1984 ; 7: 261-96.

7. Goffinet AM. The reeler gene: a clue to brain development and evolution. Int J Dev Biol 1992 ; 36 : 101-97.

8. D'Arcangelo G, Miao GG, Chen SC, Soares HD, Morgan JI, Curran T. A protein related to extracellular matrix proteins deleted in the mouse mutant reeler. Nature $1995 ; 374$ : 719-23.

9. Bar I, Lambert de Rouvroit C, Krizman DB, Royaux I, Dernoncourt C, Ruelle D, Beckers MC, Goffinet AM. A YAC contig containing the reeler locus with preliminary characterization of candidate gene fragments. Genomics 1995 ; 26 : 543-9

10. Hirotsune S, Takahara T, Sasaki N, Hirose K, Yoshiki A, Ohashi T, Kusakabe M, Murakami Y, Muramatsu M, Watanabe S, Nakao K, Katsuki M, Hayashizaki Y. The reeler gene encodes a protein with an EGF-like motif expressed by pioneer neurons. Nature Genet $1995 ; 10$ : 77-83.

11. Ogawa M, Mivata T, Nakajima K, Yagyu K, Seike M, Ikenaka K, Yamamoto H Mikoshiba $\mathrm{K}$. The reeler gene-associated antigen on Cajal-Retzius Neurons is a crucial molecule for laminar organization of cortical neurons. Neuron 1995 ; 14 : 899-912.

12. Goffinet AM. A real gene for reeler. Nature 1995 ; 374 : 675-6.

13. Rakic P, Caviness VS Jr. Cortical development: view from neurological mutants two decades later. Neuron $1995 ; 14: 1101-4$.

14. Ono K, Tomasiewicz H, Magnuson T, Rutishauser U. N-CAM mutation inhibits tangential neuronal migration and is phenocopied by enzymatic removal of polysialic acid. Neuron 1994 ; 13 : 595-609.

15. Derer P. Evidence for the occurrence of early modifications in the "glia limitans» layer of the neocortex of the reeler mutant mouse. Neurosci Lett 1979 ; 13 : 195-202

$\mathrm{m} / \mathrm{s}^{\circ} 5$, vol. 12 , mai 96 


\section{RÉFÉRENCES}

16. Derer P, Nakanishi S. Extracellular matrix distribution during neocortical wall ontogenesis in normal and reeler mice. $J$ Hirnforsch 1983 ; 24 : 209-24.

17. Derer P, Derer M. Cajal-Retzius cell ontogenesis and death in mouse brain visualized with horseradish peroxidase and electron microscopy. Neuroscience 1990 ; 36 : 839-856

18. Derer P, Derer M. Development and fate of Cajal-Retzius cells in vivo and in vitro. In: Sharma SC, Goffinet AM eds. Development of the central nervous system in vertebrates. NATO-ASI Series, vol 234. New York :

Plenum Press, 1992 : 113-29.

19. 5. Peschanski M, Rivot JP, Calvino B. Le développement du système nerveux : premières étapes. In ; Peschanski M, ed. Lexique neurobiologie. Paris : John Libbey Eurotext, 1992 : 45-6.

20. Super H, Del Rio JA, Martinez A, Soriano E. Ablation of Cajal-Retzius cells arrests neuronal migration and disrupts radial glia. 1996 (sous presse).

21. Molnar Z, Blakemore C. How do thalamic axons find their way to the cortex ? Trends Neurosci 1995 ; 18 : 389-97.

22. Chiquet-Ehrismann R. What distinguishes tenascin from fibronectin? FASEB $J$ 1990 ; 4 : 2598-604.

23. Klar A, Baldassare M, Jessel TM. F-spondin: a gene expressed at high levels in the floor plate encodes a secreted protein that promotes neural cell adhesion and neurite extension. Cell 1992 ; 69 : 95-110. 